\title{
Management of Adult Onset Familial Hemophagocytic Lymphohistiocytosis (FHL): A Dilemma
}

\section{Snehal S1, Anusree P2 , Urmi S2 ${ }^{2}$ Manisha $\mathbf{M}^{1}{ }^{*}$ and Sameer $\mathbf{M}^{2}$}

${ }^{1}$ Department of Pediatric Immunology and Leukocyte Biology, National Institute of Immunohaematology (ICMR), India

${ }^{2}$ Deenanath Mangeshkar Hospital \& Research Center, India

*Corresponding author: Manisha Madkaikar, Department of Pediatric Immunology and Leukocyte Biology, National Institute of Immunohaematology (ICMR), Parel, Mumbai-400 012, India, Tel: +91-2224138518; Fax: +91-22-24138521; Email: madkaikarmanisha@gmail.com

\section{Case Report}

Volume 2 Issue 1

Received Date: June 04, 2018

Published Date: June 18, 2018

DOI: $10.23880 / \mathrm{hij}-16000124$

\section{Abstract}

Background: Perforin gene (PRF1) mutations cause the familial form of hemophagocytic lymphohistiocytosis (HLH).

Case presentation: Here, we report a 36-year-old male with familial HLH, who was initially thought to be secondary HLH and was treated accordingly. It is only after a relapse, patient was screened for FHL which revealed perforin deficiency due to previously reported c.694C>T (p.Arg232Cys) mutation. However, till the decision of HSCT could be made, the patient succumbed to the disease.

Conclusion: Often, HLH in adults is usually attributed to an infectious etiology without a full genetic workup being undertaken. This not only causes delay in diagnosis, but also adversely affects the management of the patient. It is seen that though in few patients, the onset of HLH is delayed; the outcome remains poor as in classical severe perforin deficiency patients. Hence, it is essential to screen all the patients fitting into HLH criteria irrespective of the age of onset and identify the underlying genetic defect. Even patients with adult onset FHL have a fatal outcome unless treated with HSCT and hence prompt initiation of immunosuppressive and early HSCT is essential for the long term survival.

Keywords: FHL late onset; Perforin deficiency; Decision of management; Non-Invasive Ventilation

Abbreviations: Lymphohistiocytosis; Lymphohistiocytosis; AIDP: Acute Inflammatory Demyelinating Polyradiculoneuropathy; EBV: EpsteinBarr Virus.

\section{Introduction}

Familial hemophagocytic lymphohistiocytosis (FHL) is a severe clinical condition characterized by recurrent fever, hepatosplenomegaly, cytopenia, hepatitis and 
neurological manifestations. It is an autosomal recessive disorder with an estimated incidence of 1:50,000 live births. In 1999, Stepp, et al. [1] first documented HLH patients, with mutations in PRF1 gene (encoding perforin protein), [1]. Perforin protein is relevant for cellular cytotoxicity mechanisms and its defect results in impaired antiviral defense and dysregulation of the apoptotic mechanisms causing FHL2. The most detrimental PRF1 mutations associated with minimal or no protein expression invariably present during early infancy, with a mean age of onset of 2 months, [2]. However, there have been reports of FHL2 cases with an age at onset in teens and adulthood [3-5]. Strategies for management of these disorders, especially the decisions regarding HSCT for patients with late onset are not yet clear as there are very few case reports in the literature. Similar to infant patients, if untreated, even in adults, FHL is often fatal within a few months or even weeks.

Here we report a perforin deficiency (FHL2) case with late onset, who was initially thought to be secondary HLH and was treated accordingly. It is only after a relapse; patient was screened for FHL which revealed perforin deficiency-FHL2.

\section{Case Presentation}

In February 2015, a 36 year old male born of a nonconsanguineous marriage with no significant family history had complains of fever, fatigue and evening chills for 3 days. His hemoglobin $(\mathrm{Hb})$ was $11.7 \mathrm{~g} / \mathrm{dl}$, platelets $1,25,000 / \mathrm{mm}^{3}$ and WBC $2800 / \mathrm{mm}^{3}$. He was advised to take paracetamol, multivitamins and iron tablets. But complained of constipation and thus all the medicines were stopped. He had persistent fever and fatigue for more than 2 months which temporarily subsided on medication but reoccurred on stopping them. He was hospitalized for further investigations. His bilirubin was raised $(1.69 \mathrm{mg} / \mathrm{dl}$; reference range: $0-0.31 \mathrm{mg} / \mathrm{dl})$ revealing hepatitis. His WBC count further reduced to $1200 / \mathrm{mm}^{3}$ and platelets to $53,000 / \mathrm{mm}^{3}$. The workup for viral and bacterial infection was negative. His abdomen USG revealed hepatosplenomegaly with mild ascites and the bone marrow aspirate was suggestive of hypersplenism. After 5 days he was shifted to another hospital and bone marrow aspirate was repeated which revealed mild hypercellular marrow particle showing normoblastic to micronomroblastic maturation in erythroid series with mild relative hyperplasia and focal dyserythropoeisis, increase in number of macrophages were also noted. Occasional hemophagocytes were observed. He had raised LDH (1770U/L), triglycerides (478mg/dl), highly elevated ferritin levels $(1,00,000$ $\mathrm{ng} / \mathrm{dl})$ and reduced fibrinogen $(65 \mathrm{mg} / \mathrm{dl})$. Based on clinical manifestations (prolonged fever, hepatosplenomegaly, hepatitis) and laboratory investigations of bicytopenia (low hemoglobin and platelet count), elevated triglycerides, serum ferritin and low fibrinogen; he was diagnosed as HLH.

He was started with HLH-2004 protocol including cyclosporine (300mg-50mg tapering in 4 days), Dexamethasone (8mg to $4 \mathrm{mg}$ tapering in 4 days) and antibiotics. On treatment his fever subsided and the cell counts improved though he had persistent fatigue. However, within a month, he was again hospitalized with complains of high fever, loose motions, fatigue and pancytopenia. He was started on antibiotics; however, workup for identifying infectious agent was negative. His stool examination revealed microsporidiosis for which he was started on albendazole following which loose motions stopped. He had sudden onset of acute lumbosacral pain, non-radiating, with no neurodeficits. He was treated symptomatically for the same. He also received G-CSF in view of low cell counts and received blood and its products.

Due to relapse of the HLH symptoms, familial HLH work-up was performed for this patient, which included perforin expression, granule release assay and NK cell cytotoxicity assay by flowcytometry. Interestingly, this patient had very low perforin expression on NK cells $(5 \%$; reference range: $72 \pm 7 \%$ ), low NK cell cytotoxicity (2\%; reference range: $20 \pm 12 \%$ ) and normal granule release assay (23\%; reference range: $22 \pm 8 \%$ ). Thus indicating this patient had perforin deficiency-FHL2. Molecular analysis of PRF1 gene revealed known homozygous mutation in exon 3 (c.694C $>$ T, p.Arg232Cys). His parents and both his female siblings had partial perforin expression on NK cells (25-56\%) and were observed to be carriers of heterozygous mutation.

He was treated with cyclosporine $(100 \mathrm{mg})$ and dexamethasone (8mg) for a month and then only cyclosporine was continued. However, again after a month of stopping steroids he developed high grade fever, pancytopenia (Hb: $8.8 \mathrm{~g} / \mathrm{dl}$; WBC: $2400 / \mathrm{mm}^{3}$; Platelets: $\left.80,000 / \mathrm{mm}^{3}\right)$, elevated ferritin $(3,289 \mathrm{ng} / \mathrm{dl})$, borderline high triglycerides $(227 \mathrm{mg} \%)$ and high bilirubin $(3.76 \mathrm{mg}$ $\%$ ). Thus dexamathasone ( $4 \mathrm{mg}$ ) was started again and the fever subsided immediately. He was on maintenance with cyclosporine and pulse doses of steroids for around 4 months and then it was tapered slowly. He was then off steroids for more than 6 months and was leading a 
routine life, thus making the decision of HSCT a dilemma. However, in mid June 2017, approximately a year post stopping treatment, he again had a relapse.

This time he presented with headache, left limb swelling, loss of appetite and yellowish coloration of sclera. He was neurologically conscious and oriented but CT scan revealed mild cerebral and cerebellar atrophy. Routine laboratory investigations were suggestive of liver and renal dysfunction and persistent hyperkalemia. On the second day of admission he had severe tachypnea and tachycardia and required non-invasive ventilation (NIV) support. Inspite of prompt supportive treatment, patient succumbed to the disease due to multiple organ failure and septic shock.

\section{Discussion and Conclusion}

In literature, perforin deficiency has been reported to account for $20-40 \%$ of FHL patients depending on the ethnic origin, and these patients with perforin deficiency present early in life and have severe clinical manifestations [1]. Also majority of the literature on the clinical spectrum and natural course of FHL derives from reports of children and this lead to the common belief that FHL is restricted to children or infants, and does not pertain to adult patients. However, there have been reports of FHL2 cases with an age of onset in teens and adulthood, with few patients presenting in sixth and seventh decade of life, $[1,3,5,6]$. The concept that later onset of HLH is possible has been repeatedly brought to the attention of adult haematologists. Nevertheless, adult patients are at most considered as potentially affected by the acquired form of HLH, rather than by FHL. This leads to misdiagnosing or delay in diagnosis of this life threatening syndrome.

In the case reported here, the patient had onset of the disease at 36years of age and had no significant family history and thus was considered as secondary HLH. He was clinically fitting into HLH diagnostic criteria and hence was initially started on HLH-2004 protocol. It was only when he had relapse of disease when on therapy, he was worked-up for FHL, wherein he was found to be perforin deficient. The genetic study of PRF1 gene revealed homozygous mutation in exon 3 (c.694C>T, p.Arg232Cys). This mutation changes positively charged arginine to non-charged polar amino acid cysteine at position 232, which lies in the MACPF domain of perforin protein, essential for lytic activity. This mutation has been previously reported in heterozygous state in two cases.
In first case, the patient presented at 6 years of age and had severe clinical manifestations. This patient had compound heterozygous mutations (g.1182insT; p.Gly394fsX and c.694C>T, p.Arg232Cys). Inspite of late onset, this patient expired within 15days of diagnosis, even before starting the therapy [3]. In another case, this mutation was in heterozygous state along with other heterozygous mutation (g.4246_4247insTG; p.His398CysfsX23). Presented at 6 years of age with acute inflammatory demyelinating polyradiculoneuropathy (AIDP) associated with a fatal haemophagocytic lymphohistiocytosis (HLH). The patient developed progressive weakness of the lower limbs in the context of a picture of infectious mononucleosis and Epstein-Barr virus (EBV) infection. After an apparent improvement, a fulminant hepatic failure and pancytopenia ensued, leading to death [7]. In both these cases, inspite of compound heterozygosity of mutation in PRF1 gene and late onset, once the disease developed, it was fatal.

Clementi, et al. [4] in 2002 reported 2 siblings sharing the same mutations in the PRF1 gene, who developed HLH at 22 and 21 years, respectively. One of the siblings required bone marrow transplant (BMT), whereas the other is continued on cyclosporine and was symptomatic when the report was written [4]. Mancebo, et al. [5] in 2006 reported a 49-year-old male patient, homozygous for $\mathrm{A} 91 \mathrm{~V}$ in the perforin gene, who developed HLH with a tuberculosis infection. In 2007, Nagafugi, et al. [6] reported a 62-year-old male patient with decreased but residual perforin activity and harbor compound heterozygous mutation of PRF1 (1090-1091delCT and $916 \mathrm{G} \rightarrow \mathrm{A}$ ). This patient was on steroid treatment and was asymptomatic. Recently, Zhang et al. reported twenty-five adult patients with HLH possessed genetic mutations in North America and Sieni, et al. [8] described eleven adult FLH in Italy $[8,9]$. Irrespective of the mutations, the outcome of these patients differed. In the report by Sieni, 2 patients died even before starting the protocol while 3 died after initiating the protocol, 3 patients had HSCT rejection and succumbed to the disease, 3 patients are on therapy and awaiting HSCT and only 1 patient had successful SCT.

In our previous report [10] in 2013 we reported 4 cases of atypical presentations and late onset of disease wherein 2 patients succumbed to the disease before initiating the therapy whereas other 2 had multiple relapses on tapering the therapy. However, later 1 of these patients expired due to severe disease and the other lost follow-up. 
Also, in the case presented here, the patient survived for more than 2 years after the onset of disease. Inspite of having several relapses and identification of genetic defect, the fact that otherwise he was leading a normal routine life and the potential HSCT donors (his sisters and parents) were carrier of the mutation, made the decision of transplant difficult. Thus it can be interpreted that in adults, FHL may present with acute onset of full-blown $\mathrm{HLH}$, or as a more insidious illness with recurrent bouts of nonspecific symptoms of HLH which may resolve spontaneously or with steroid treatment alone or may require $\mathrm{HSCT}$.

With the number of case studies reporting the adult FHL patients, it is now understood that patients with a genetic basis for HLH can sometimes remain asymptomatic until adolescence or even adulthood [4-6]. Mutations causing reduced or residual perforin expression and NK cell function could be the basis of HLH that is dormant until triggered by external factors such as infection or other environmental stresses [5,9]. However, the exact mechanism behind such a delay remains unknown and thus the decision of treatment remains a dilemma.

To conclude, it is important that the general physicians and pediatricians are aware of the diagnosis of FHL and all the patients suspected for HLH, irrespective of age of onset should be screened by FHL workup. Identification of a genetic defect in patients with HLH has diagnostic, prognostic, and therapeutic implications and should be pursued whenever possible. Despite frequent reports on variation in the age at onset within each family, asymptomatic sibs (including potential stem cells donors) cannot be safely defined as unaffected, unless their genetic status for HLH is assessed. Lack of this information may risk BMT from an affected donor in a pre-symptomatic phase.

- Informed consent: Written informed consent was obtained from parents before writing this manuscript.

- Ethical clearance: The study was approved by a Departmental Review Board.

- Conflict of interest: None of the authors has any potential financial conflict of interest related to this manuscript.

\section{References}

1. Stepp SE, Dufourcq-Lagelouse R, Le Deist F, Bhawan S, Certain S, et al. (1999)Perforin gene defects in familial hemophagocytic lymphohistiocytosis. Science 286(5446): 1957-1959.

2. Trizzino A, zur Stadt U, Ueda I, Risma K, Janka G, et al. (2008) Genotype-phenotype study of familial haemophagocytic lymphohistiocytosis due to perforin mutations. J Med Genet 45(1): 15-21.

3. Clementi R, zur Stadt U, Savoldi G, Varoitto S, Conter $V$, et al. (2001) Six novel mutations in the PRF1 gene in children with haemophagocytic lymphohistiocytosis. J Med Genet 38(9): 643-646.

4. Clementi R, Emmi L, Maccario R, Liotta F, Moretta L, et al. (2002) Adult onset and atypical presentation of hemophagocytic lymphohistiocytosis in siblings carrying PRF1 mutations.," Blood 100(6): 2266-2267.

5. Mancebo E, Allende LM, Guzmán M, Paz-Artal E, Gil J, et al. (2006) Familial hemophagocytic lymphohistiocytosis in an adult patient homozygous for $\mathrm{A} 91 \mathrm{~V}$ in the perforin gene, with tuberculosis infection. Haematologica 91(9): 1257-1260.

6. Nagafuji $\mathrm{K}$, Nonami A, Kumano $\mathrm{T}$, Kikushige $\mathrm{Y}$, Yoshimoto G (2007) Perforin gene mutations in adultonset hemophagocytic lymphohistiocytosis. Haematologica 92(7): 978-981.

7. Del Giudice E, Savoldi G, Notarangelo LD, Di Benedetto L, Manganelli F (2003) Acute inflammatory demyelinating polyradiculoneuropathy associated with perforin-deficient familial haemophagocytic lymphohistiocytosis. Acta paediatr 92(3): 398-401.

8. Elena Sieni, Valentina Cetica, Andrea Piccin, Filippo Gherlinzoni, Ferdinando Carlo Sasso, et al. (2012) Familial Hemophagocytic Lymphohistiocytosis May Present during Adulthood: Clinical and Genetic Features of a Small Series. PLoS ONE 7(9): e44649.

9. Zhang K, Jordan MB, Marsh RA, Johnson JA, Kissell D (2011) Hypomorphic mutations in PRF1, MUNC13-4, and STXBP2 are associated with adult-onset familial HLH. Blood 118(22): 5794-5798.

10. Mhatre S, Madkaikar M, Jijina F, Ghosh K (2014) Unusual Clinical Presentations of Familial Hemophagocytic Lymphohistiocytosis Type-2. J Pediatr Hematol Oncol 36(8): 524-527. 
Haematology International Journal

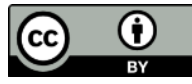

Manisha M, et al. Management of Adult Onset Familial Hemophagocytic Lymphohistiocytosis (FHL): A Dilemma. Haematol Int J 2018, 2(1): 000124. 